\title{
BMJ Global Health Impact of a community-based package of interventions on child development in Zambia: a cluster-randomised controlled trial
}

\author{
Peter C Rockers, ${ }^{1}$ Günther Fink, ${ }^{2}$ Arianna Zanolini, ${ }^{3}$ Bowen Banda, ${ }^{4}$ \\ Godfrey Biemba, ${ }^{4}$ Cierra Sullivan, ${ }^{5}$ Simon Mutembo, ${ }^{6}$ Vichaels Silavwe, ${ }^{6}$ \\ Davidson $\mathrm{H} \mathrm{Hamer}^{4}$
}

To cite: Rockers PC, Fink G, Zanolini A, et al. Impact of a community-based package of interventions on child development in Zambia: a cluster-randomised controlled trial. BMJ Global Health 2016;1:e00104.

doi:10.1136/bmjgh-2016000104

- Additional material is available. To view please visit the journal (http://dx.doi.org/ 10.1136/bmjgh-2016000104).

Received 9 June 2016 Revised 1 October 2016 Accepted 3 October 2016

CrossMark

For numbered affiliations see end of article.

Correspondence to

Peter C Rockers;

prockers@bu.edu

\section{ABSTRACT}

Background: Community-based programmes are a critical platform for improving child health and development. We tested the impact of a communitybased early childhood intervention package in rural Zambia.

Methods: We conducted a non-blinded cluster randomised controlled trial in Southern Province, Zambia. 30 clusters of villages were matched based on population density and distance from the nearest health centre, and randomly assigned to intervention (15 clusters and 268 caregiver-child dyads) or control (15 clusters and 258 caregiver-child dyads). Caregivers were eligible if they had a child aged 6-12 months at baseline. In intervention clusters, health workers screened children for infections and malnutrition, and invited caregivers to attend fortnightly group meetings covering a nutrition and child development curriculum. 220 intervention and 215 control dyads were evaluated after 1 year. The primary outcomes were stunting and INTERGROWTH-21st neurodevelopmental assessment (NDA) scores. Weight-for-age and height-for-age z-scores based on WHO growth standards were also analysed.

Secondary outcomes were child illness symptoms, dietary intake and caregiver-child interactions based on self-report. Impact was estimated using intentionto-treat analysis.

Results: The intervention package was associated with a $0.12 \mathrm{SD}$ increase in weight-for-age $(95 \% \mathrm{Cl}$ -0.14 to 0.38 ), a $0.15 \mathrm{SD}$ increase in height-for-age $(95 \% \mathrm{Cl}-0.18$ to 0.48$)$ and a reduction in stunting (OR $0.68 ; 95 \% \mathrm{Cl} 0.36$ to 1.28 ), whereas there was no measurable impact on NDA score. Children receiving the intervention package had fewer symptoms, a more diverse diet and more caregiver interactions.

Conclusions: In settings like Zambia, communitybased early childhood programmes appear to be feasible and appreciated by caregivers, as evidenced by high rates of uptake. The intervention package improved parenting behaviours and had a small positive, though statistically insignificant, impact on child development. Given the short time frame of the project, larger developmental impact is likely if differential parenting behaviours persist.

\section{Key questions}

What is already known about this topic?

- Recent research has demonstrated that community-based interventions can improve child development outcomes. However, how to best package and deliver interventions in lowresource settings like Zambia remains unclear.

What are the new findings?

- This is the first trial to assess the impact of a package of early childhood development interventions in Zambia, a low-income country with a tremendous burden of child illness and malnutrition.

- Our findings, taken together with the available evidence, suggest that community-based early childhood programmes are feasible and appreciated by caregivers in settings like Zambia.

- The intervention package that we tested showed a strong positive impact on parenting behaviours. While the impact we observe on child development was small and not statistically significant at the end of the 1-year study period, larger developmental impact is likely if differential parenting behaviours persist.

\section{Recommendations for policy}

- Regular household visits coupled with group meetings appear to positively impact caregiver behaviours. In settings like Zambia, where a substantial proportion of children fail to reach their developmental potential, these interventions hold great promise for improving child health and welfare.

- Improving parenting behaviours may not immediately yield large improvements in developmental outcomes; continued and sustained parental and governmental efforts are likely needed to allow children to fully reach their developmental potential.

Trial registration number: NCT02234726; Results. 


\section{INTRODUCTION}

Globally, a large number of children continue to struggle to reach their developmental potential. According to most recent estimates, $30 \%$ of children under age 5 are stunted and over $40 \%$ do not reach their full developmental potential. ${ }^{1}{ }^{2}$ Chronic undernutrition, high rates of infectious diseases and lack of stimulation in the home environment early in life are associated with poor language and cognitive development, with persistent effects into adulthood. ${ }^{3}$ Early childhood interventions have been shown to lessen the negative impact of these exposures and improve developmental outcomes. ${ }^{4} 5$ Improved screening and treatment for infections can positively impact physical growth and cognitive development in children, ${ }^{6}$ and teaching parents play-based activities to stimulate children has been shown to improve language, motor function and cognitive development. ${ }^{7-9}$ Given the multifaceted developmental risks faced by children, the need for multisectoral intervention packages has been increasingly recognised in recent years. $^{10}$

Community-based platforms are currently the primary strategy to deliver early childhood services to rural populations in developing countries. In Jamaica, the provision of early childhood services by community health workers (CHWs) as part of weekly or fortnightly home visits has been shown to be feasible and effective. $^{7} 8$ In Pakistan, CHWs have successfully implemented home-based nutrition and stimulation interventions. ${ }^{11}$ Community-based parenting groups have also been shown to be an effective and low-cost platform for delivering child health messages. ${ }^{12-15}$ In Cambodia, a state-sponsored programme that delivers early childhood services through a community-based group meeting model has shown initial positive results. ${ }^{16}$ Finally, in Uganda, a peer-based service delivery platform has been effective at improving parenting practices and child development outcomes. ${ }^{9}$ While community-based models appear promising, the best methods for packaging and delivering early childhood interventions in resource-limited settings like Zambia remain unclear, limiting their implementation and large-scale uptake.

We conducted a cluster randomised controlled trial to test the impact of a package of community-based early childhood interventions in a rural area of Southern Province, Zambia. As part of the trial, households in communities randomised to the intervention group received fortnightly visits from a local health worker and were invited to attend a fortnightly group meeting where caregivers were taught a diverse curriculum with content related to child development. The primary outcomes were child physical growth and neurodevelopment. The findings from this study should inform future child development policy in Zambia, a country that is attempting to address high rates of child malnutrition and infectious diseases through community-based strategies.

\section{METHODS}

\section{Study design and setting}

The study was a cluster randomised controlled trial. It was conducted in Choma and Pemba districts in Southern Province, Zambia. Rural subsistence farmers comprise the majority of the population in this area. According to the latest 2013 Zambia Demographic Health Survey (DHS), 37\% of children under age 5 in Southern Province are stunted and 14\% are severely stunted; $13 \%$ are underweight. ${ }^{17}$ Infections are highly prevalent: $21 \%$ of children under 5 were reported with a fever in the 2 weeks preceding the survey, and $18 \%$ with diarrhoea. Parents sought treatment for $74 \%$ of recent child fevers, almost exclusively at the nearest public health centre.

\section{Participants}

A random sample of households was selected for study recruitment using a multistage sampling procedure. First, five rural health centres (RHCs) were purposefully selected as study sites. The study team had worked with the staff of the selected RHCs as part of a previous research project. ${ }^{18}$ Each RHC covers a population of $\sim 10000$ residing in 6-10 health zones. Within each RHC catchment area, six health zones were randomly selected. All villages within each selected health zone were then listed, along with estimates of the number of eligible children in each village (see eligibility criteria below), based on health facility records. Villages were randomly selected with probability proportional to size to reach the target sample of 18 eligible children in each health zone size, and 540 children in total. Villages with one or fewer eligible children were excluded from consideration due to the infeasibility of implementing the group meeting component of the intervention package in these villages.

The primary household eligibility criterion for study inclusion was having a child aged between 6 and 12 months at the time of enrolment. Previous research suggests that, due to the cumulative nature of deficits in child development, intervening as early as possible (eg, in the first 1000 days) is likely to be most effective. ${ }^{4} 19$ Eligibility was also restricted to female caregivers aged 15 years and older. Selected villages were visited, and all eligible households were listed and recruited into the study. Informed consent was obtained from all participating caregivers prior to data collection.

The study was powered to detect a $0.5 \mathrm{SD}$ increase in height-for-age $\mathrm{z}$-score (HAZ) in the intervention arm relative to the control arm with $80 \%$ power, assuming 30 clusters of equal size and an intracluster correlation coefficient of 0.1 . The final sample size provided enough power to detect a $0.5 \mathrm{SD}$ increase in Neurodevelopmental Assessment (NDA) z-score with $90 \%$ power.

\section{Randomisation and blinding}

Households were randomised at the level of the health zone (cluster) to the intervention or the control group 
by the study team prior to baseline enrolment. To increase statistical power and reduce the threat of potential confounding, prior to randomisation health zones were matched in pairs within the RHC catchment area based on two factors: distance to the health centre and number of villages (as a proxy for population density). Within each matched pair, one zone was randomly selected to receive the intervention using a computerbased random number generator. Group assignment was blinded from all data collectors, though the survey included questions about mothers groups. Blinding of participants was not possible due to the nature of the intervention.

\section{Procedures}

Intervention households were visited fortnightly by a community-based health worker employed full time by the project which we refer to as Child Development Agents (CDAs), who screened and referred children for infections and acute malnutrition, and provided parents with reminders to use routine child health services, including immunisations. Caregivers were also invited to attend fortnightly group meetings, where they were taught a diverse curriculum with content on cognitive stimulation and play practices, child nutrition and cooking practices, and self-care for good mental health. Household visits and group meetings were held throughout the 1-year study intervention period.

At the start of the study, 10 women were recruited from local communities to serve as CDAs. They were selected from a pool of candidates who had worked with members of the study team on a previous study conducted in the same communities, and were originally identified through consultation with community leaders and neighbourhood health committees. ${ }^{18}$ All CDAs had previous experience providing community-based health services, some as part of the formal health system. All had at least a ninth grade education, the ability to read and write in English and Tonga (the local language), a reputation for being well respected in their communities and past child healthcare experience. Prior to starting fieldwork, all CDAs underwent a 5-day training during which they received a refresher on diagnosing child illness and supporting child development, learnt the protocols for the home visits and were trained on how to facilitate and support the group meetings.

CDAs conducted fortnightly household visits during which they completed three main activities: (1) screening and referral for symptoms of infectious disease, including malaria, diarrhoea and pneumonia; (2) screening and referral for acute malnutrition based on midupper arm circumference ${ }^{20}$ and (3) encouragement of the use of routine care services for children, for example, immunisations, growth monitoring and vitamin A supplementation. Each CDA was responsible for visits to around 25 households.

In addition to conducting household visits, CDAs also facilitated fortnightly group meetings in intervention communities. A few meetings were held in each intervention cluster, in locations chosen by the community to minimise the burden on caregivers traveling from nearby villages. Each group meeting was run by a local 'head mother', selected by the community itself, and CDAs did not actually attend. Rather, CDAs regularly met with all head mothers prior to each round of meetings, and provided training and resources according to the planned curriculum for that rounds' meeting. Meetings combined locations were established to minimise the travel burden took place. Each meeting round focused on a different topic, including parenting skills, child nutrition and cooking demonstrations, forms of play, cognitive stimulation and language development activities. The curriculum included elements adapted from existing child development programmes. ${ }^{21}{ }^{22}$ All female caregivers in study communities with children aged under 5 years were invited to attend meetings, not only those who were enrolled in the study. It was decided based on local consultation that it would not have been appropriate to invite male caregivers to attend group meetings. Children accompanied caregivers to meetings, and many aspects of the curriculum involved interactions with children. In total, 20 rounds of meetings were held during the study period.

Data were collected from two main sources: forms completed by CDAs during household visits, and household surveys conducted at baseline (August/September 2014) and endline (September/October 2015). CDA's visit forms provide key information to track the implementation of the intervention package, including the volume of $\mathrm{CDA}$ home visits. Caregiver attendance at group meetings was also assessed during CDA visits by asking caregivers if they attended a meeting during the previous 2 weeks; missing data on meeting attendance were imputed. Household visit forms were developed and administered using the TeleForms system, which enables hand-written text to be translated to computer readable files. The system includes a data verification system, eliminating the need for multiple data entry. ${ }^{23}$ Completed forms were collected from CDAs at regular intervals and immediately scanned into a computer database, and these data were quality checked each month.

\section{Outcomes}

The primary outcomes were children's physical and cognitive development. Children's height and weight were measured both at baseline and endline using standard anthropometric assessments kits. These data were converted into z-scores using the WHO's Multicentre Growth Standards. ${ }^{24}$ Parental reports of child gross motor function and cognition were captured using the Saving Brains Early Child Development Scale. Finally, at the endline study, children were administered the INTERGROWTH-21st NDA tool to assess cognitive and socioemotional development. The NDA is a multidimensional, mixed methodology, screening package that includes components of the Bayley Scales of Infant 
Development, the Rapid Neurodevelopmental Assessment, the Malawi Developmental Assessment, the Griffiths Mental Development Scale and the Child Behavior Checklist. The package includes 53 items: 30 cognitive, language and motor items; 6 behavioural items and 17 items measuring attention and emotional reactivity. It was developed for use in low-resource settings like Zambia, was designed to be simple and easy to administer by trained survey staff, and has recently been validated in several developing countries. ${ }^{25}$

Key secondary outcomes, measured at baseline and endline, included the prevalence of child illness symptoms-fever, diarrhoea and cough-in the previous 2 weeks; child diet in the previous 24 hours and parentchild interactions using the six-item Multiple Indicator Cluster Survey module. ${ }^{26}$ A standardised diet diversity score was constructed based on the number of food groups children had consumed the previous day, as an indicator of micronutrient adequacy as per the method described in Steyn et al. ${ }^{27}$ Household demographics and asset information, as well as caregiver mental health assessed using the 20-item WHO Self-Reporting Questionnaire (SRQ), ${ }^{28}$ were also collected.

\section{Statistical analysis}

We describe the study population and compare baseline characteristics between the intervention and control groups, including those interviewed at endline and those lost to follow-up. Next, we describe the implementation and uptake of the study intervention package with histograms of the number of household visits and attendance at group meetings. Then, we investigate differences between the control and interventions groups on the primary and secondary outcomes of interest. All SEs are clustered to account for the study design. Impact was assessed using intention-to-treat analysis. All analyses were conducted using Stata statistical software
(StataCorp. Stata Statistical Software: Release 14. College Station, TX: StataCorp LP, 2015). This trial was registered on ClinicalTrials.gov prior to baseline data collection, number NCT02234726.

\section{RESULTS \\ Study population}

Thirty clusters were randomly assigned to intervention (15 clusters and 268 caregivers-child dyads) or control (15 clusters and 258 caregiver-child dyads). At the end of the trial (last visit 2 October 2015), 220 caregiverchild dyads (82\%) remained in the intervention group, whereas 215 dyads $(83 \%)$ remained in the control group (figure 1). The difference in attrition rate between the treatment groups was not statistically significant $(p=0.76)$. The majority of households lost to follow-up moved from the study area and could not be located. Baseline characteristics of enrolled caregivers and children were similar in the intervention and control groups (table 1).

Children aged, on average, 8 months at the start of the study, with a relatively uniform distribution within the range of eligibility (6-12 months). Height and weight were well below the international reference median, with a mean HAZ of -1.5 and a mean weight-for-age $z$-score (WAZ) of -0.3 at baseline. Study caregivers aged, on average, 27 years at baseline and just less than half had completed primary school.

\section{Programme reach}

In total, there were 23 rounds of household visits over the study period. Around one-quarter of households were visited in every round and $68 \%$ of households were visited at least 20 times (figure 2A). On average, households received 19 visits from CDAs. One main reason for missed visits was the rainy season making it impossible to access the most remote villages. One

Figure 1 Trial profile.

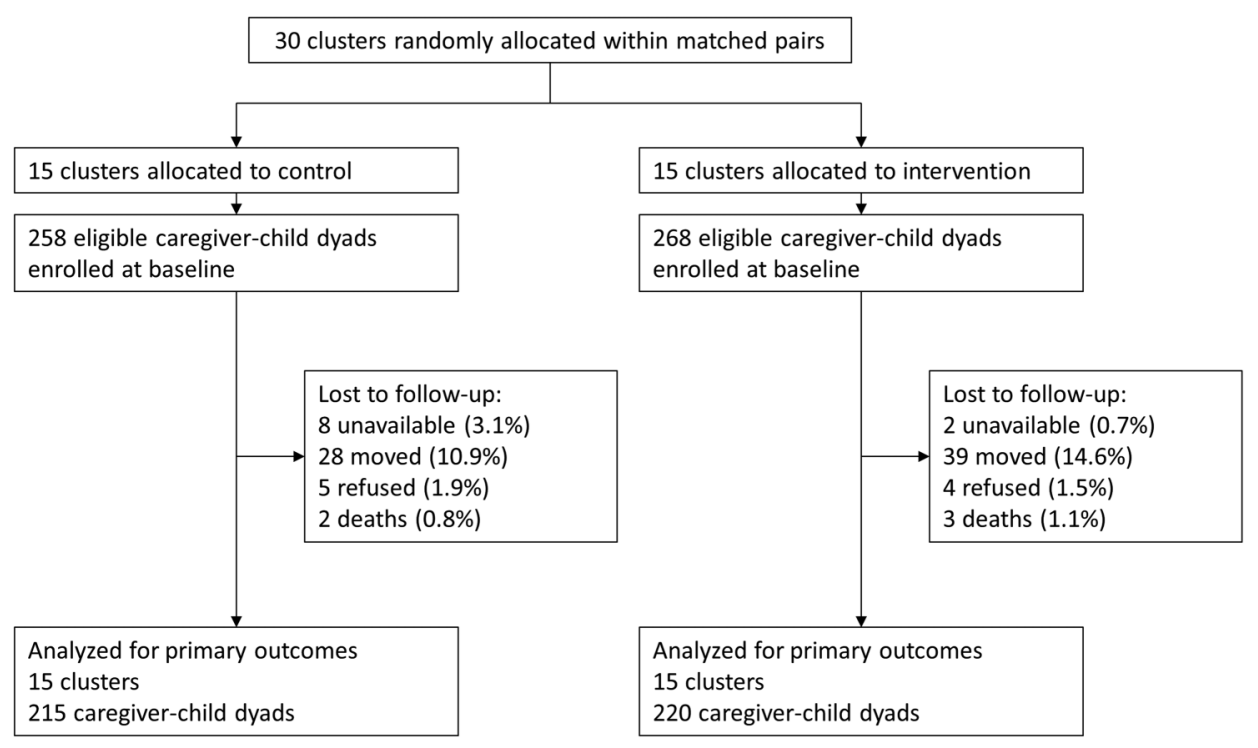


Table 1 Baseline characteristics of the study participants

\begin{tabular}{|c|c|c|c|c|c|c|}
\hline & \multicolumn{3}{|l|}{ Control } & \multicolumn{3}{|l|}{ Intervention } \\
\hline & $\begin{array}{l}\text { All enrolled } \\
(n=258)\end{array}$ & $\begin{array}{l}\text { Remaining at } \\
\text { endline } \\
(n=215)\end{array}$ & $\begin{array}{l}\text { Lost to follow-up } \\
(n=43)\end{array}$ & $\begin{array}{l}\text { All enrolled } \\
(n=268)\end{array}$ & $\begin{array}{l}\text { Remaining } \\
\text { at endline } \\
(n=220)\end{array}$ & $\begin{array}{l}\text { Lost to } \\
\text { follow-up } \\
(n=48)\end{array}$ \\
\hline \multicolumn{7}{|c|}{ Child characteristics at baseline } \\
\hline Age (months) & $8.50(2.03)$ & $8.49(2.05)$ & $8.53(1.98)$ & $8.74(2.17)$ & $8.70(2.17)$ & $8.92(2.18)$ \\
\hline Female & $0.51(0.50)$ & $0.52(0.50)$ & $0.44(0.50)$ & $0.47(0.50)$ & $0.49(0.50)$ & $0.38(0.49)$ \\
\hline Stunted & $0.35(0.48)$ & $0.32(0.47)$ & $0.54(0.50)$ & $0.40(0.49)$ & $0.39(0.49)$ & $0.46(0.50)$ \\
\hline $\begin{array}{l}\text { Weight-for-age } \\
\text { (z-score) }\end{array}$ & $-0.31(1.38)$ & $-0.29(1.45)$ & $-0.44(1.00)$ & $-0.45(1.38)$ & $-0.47(1.39)$ & $-0.38(1.38)$ \\
\hline Underweight & $0.12(0.32)$ & $0.13(0.33)$ & $0.07(0.26)$ & $0.15(0.35)$ & $0.15(0.35)$ & $0.15(0.36)$ \\
\hline $\begin{array}{l}\text { Cough in the } \\
\text { previous } 2 \text { weeks }\end{array}$ & $0.47(0.50)$ & $0.51(0.50)$ & $0.24(0.43)$ & $0.40(0.49)$ & $0.41(0.49)$ & $0.35(0.48)$ \\
\hline \multicolumn{7}{|c|}{ Caregiver characteristics at baseline } \\
\hline Age (years) & $27.57(8.94)$ & $27.92(9.32)$ & $25.81(7.09)$ & $26.98(7.75)$ & $27.45(7.79)$ & $24.66(8.34)$ \\
\hline $\begin{array}{l}\text { Completed primary } \\
\text { school }\end{array}$ & $0.46(0.50)$ & $0.44(0.50)$ & $0.53(0.50)$ & $0.47(0.50)$ & $0.45(0.50)$ & $0.56(0.50)$ \\
\hline $\begin{array}{l}\text { Mental health (SRQ } \\
\text { z-score) }\end{array}$ & $0.13(1.08)$ & $0.16(1.07)$ & $0.00(1.12)$ & $-0.13(0.90)$ & $-0.11(0.90)$ & $-0.22(0.93)$ \\
\hline $\begin{array}{l}\text { Household wealth } \\
\text { quintile }\end{array}$ & $2.85(1.42)$ & $2.87(1.42)$ & $2.77(1.43)$ & $3.13(1.41)$ & $3.21(1.41)$ & $2.73(1.33)$ \\
\hline
\end{tabular}

Data are mean (SD). Child height-for-age and weight-for-age z-scores are normalised to WHO standards. Stunted is defined as height-for-age $z$-score $\leq-2$. Underweight is defined as weight-for-age $z$-score $\leq-2$. Caregiver SRQ $z$-scores are normalised within the study population. Lower $S R Q$ z-score indicates better mental health.

SRQ, Self-Reporting Questionnaire.

CDA was also found to be not making scheduled visits for a period of 2 months; this CDA was replaced and regular household visits for the affected communities restarted.

There were 20 rounds of group meetings held by community-based mother heads during the study period. While few caregivers attended all of the meetings, the majority (89\%) attended 10 or more meetings, whereas only four caregivers $(2 \%)$ attended $<5$ meetings (figure 2B). On average, enrolled caregivers attended 14 group meetings. Average overall attendance at meetings was around 10, including study participants as well as other caregivers from the community with young children.

\section{Impact of the intervention package on child health and development}

The intervention package was associated with a 0.12 SD increase in WAZ (95\% CI -0.14 to 0.38 ) and a 0.15 SD increase in HAZ (95\% CI -0.18 to 0.48 ; table 2). While the intervention was also associated with reduced odds of stunting (OR 0.68 ; $95 \%$ CI 0.36 to 1.28 ), children in the intervention and control groups exhibited low stunting rates at endline (23\% and $25 \%$, respectively). In terms of child development outcomes, the intervention was associated with a $0.11 \mathrm{SD}$ increase in child motor function (95\% CI -0.11 to 0.33 ), a $0.11 \mathrm{SD}$ increase in cognition $(95 \%$ CI -0.09 to 0.32 ) and a 0.10 SD decrease in NDA score (95\% CI -0.35 to 0.15 ).

Large significant impact was found for caregiver behaviour and secondary study outcomes. Caregivers in the intervention groups more often reported engaging with their children in nearly all measured activities in the previous 3 days, including reading to their child (OR 3.58; $95 \%$ CI 2.32 to 5.52 ), signing songs with their child (OR 3.38; 95\% CI 1.85 to 6.18 ) and playing with their child (OR 2.59; 95\% CI 1.64 to 4.07). Caregivers in the intervention group had lower SRQ z-scores at endline, though this difference was not statistically significant in the adjusted model ( $\beta-0.13 ; 95 \%$ CI -0.38 to 0.11 ). Children in the intervention group had significantly lower odds of fever (OR 0.68; 95\% CI 0.47 to 0.98 ) and a trend towards lower odds of diarrhoea (OR 0.65; $95 \%$ CI 0.41 to 1.03 ) and cough (OR 0.63; $95 \%$ CI 0.36 to 1.07 ) in the previous 2 weeks (table 3 ). Finally, children in the intervention group had a significantly more diverse 

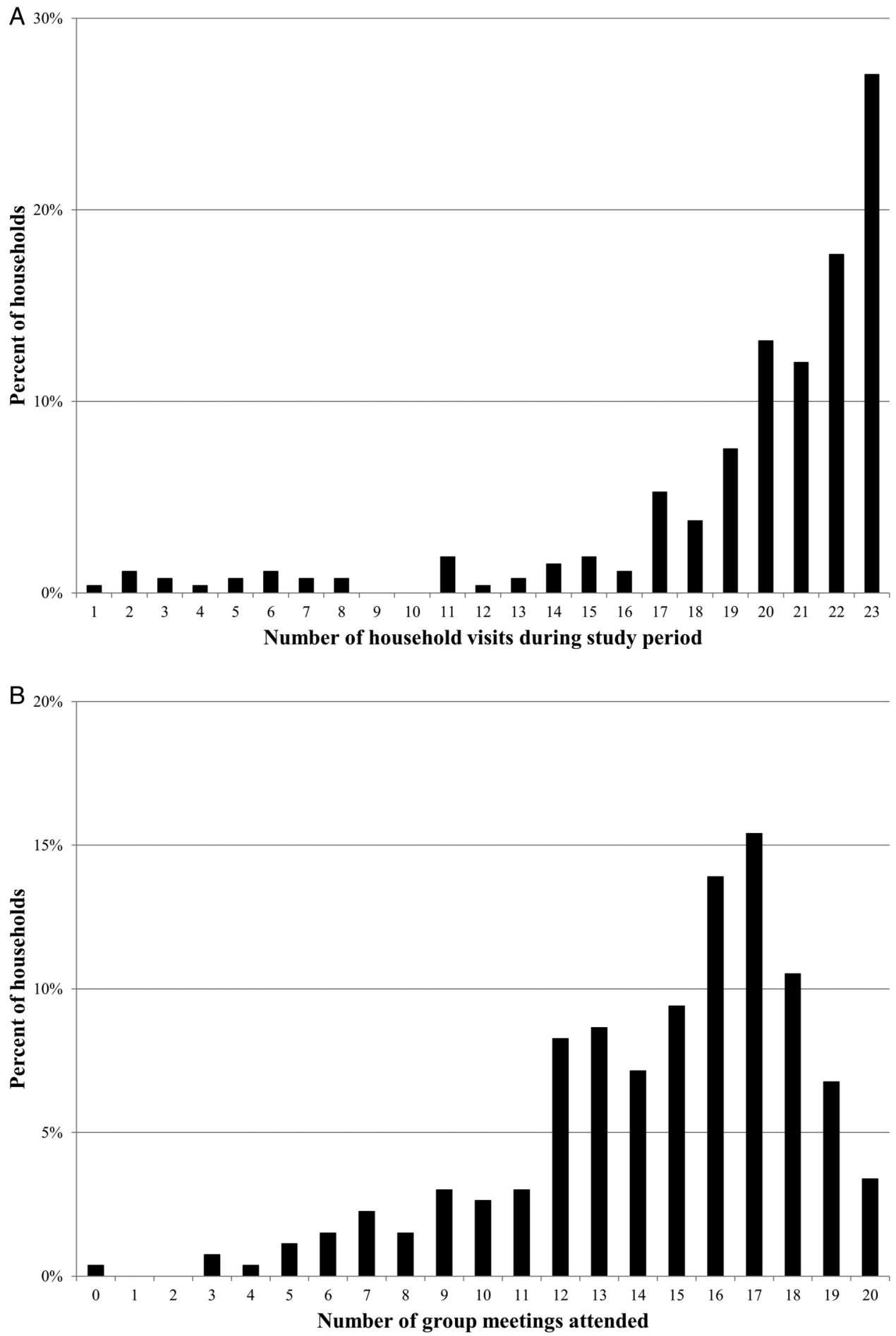

Figure 2 (A) Household visits. (B) Attendance at group meetings.

$\operatorname{diet}(\beta 0.24 ; 95 \%$ CI 0.08 to 0.41$)$ and were more likely to have consumed meats (OR 1.29; $95 \%$ CI 0.77 to 2.14 ) and non-meat proteins (OR 1.43; 95\% CI 0.88 to 2.31) like nuts and beans in the preceding 24 hours. Children in the intervention group were also more likely to have consumed roller meal (an unrefined and protein-rich version of the local maize staple) and less likely to have consumed breakfast meal (a more refined and less protein-rich version of maize). In online supplementary appendix 1, we present primary and secondary outcomes stratified by attendance at group meetings, and find that results look very similar across groups.

\section{DISCUSSION}

Our results suggest that the intervention package is feasible, with relatively few difficulties encountered in its implementation, and all services well received by 
Table 2 Impact of the intervention package on child development

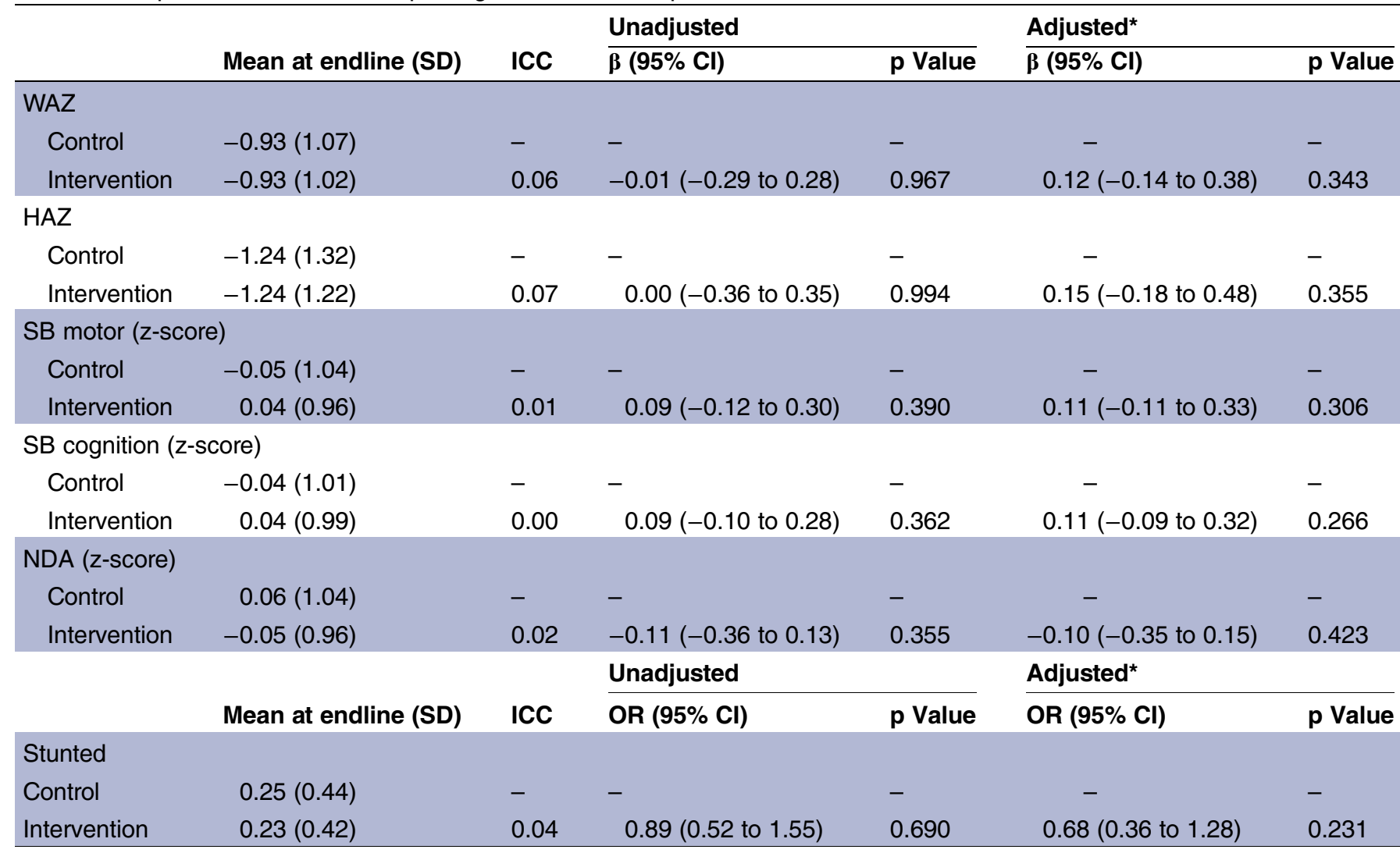

*Controlling for: child's age, gender, siblings, baseline HAZ, baseline WAZ, baseline WHZ, baseline motor function z-score, caregiver's age; education and baseline SRQ z-score, baseline caregiver-child interaction score, household wealth, household inhabitants and gender of household head.

HAZ, height-for-age z-score; ICC, intracluster correlation coefficient; NDA, Neurodevelopment Assessment; SB, Saving Brains Early Child Development Scale; SRQ, Self-Reporting Questionnaire; WAZ, weight-for-age z-score; WHZ, Weight-for-height z-score.

caregivers. Despite no material incentive for doing so, a large proportion of caregivers attended a majority of group meetings. The intervention had a strong positive impact on key parenting behaviours related to child development, including caregiver-child interaction and child nutrition. While child growth and development outcomes mostly moved in the expected direction, none of the observed changes was statistically significant.

There are several potential explanations for the modest impact on developmental measures despite the pronounced shifts in parental behaviour. The behavioural changes we observed in the intervention group may not have been sufficient to yield large improvements in child development. Many of the observed changes in behaviour were rather substantial, making a complete absence of developmental impact unlikely, but improvements may take more time to fully materialise. Given that direct assessments of children below age 2 are challenging in low-income settings like Zambia, it is also possible that the study instruments were not sensitive enough to detect differential cognitive trajectories. Larger differentials would likely emerge with longer term follow-up.

One of the most surprising findings of the trial is the large overall improvement in stunting in treatment and control groups. At baseline, when children aged $6-12$ months, the prevalence of stunting was $40 \%$ and $35 \%$ in the intervention and control groups, respectively. At endline, when children aged 18-24 months, these rates were $23 \%$ and $25 \%$, respectively. This is substantial improvement in an age range generally characterised by pronounced growth faltering: the prevalence of stunting among children aged 18-24 months in Zambia is 54\% according to the most recent DHS. ${ }^{29} \mathrm{HAZ}$ among study children in both groups at baseline was in line with DHS averages for that age range. There is no evidence of a child nutrition programme in the study area that could account for this finding. Rather, anecdotal evidence collected after the completion of the trial suggests that caregivers in control villages may have been motivated by the study itself to focus on their children's health and nutrition. If this did occur, the impact of the intervention package might be substantially underestimated.

There were several key limitations to this work. First, rather than delivering the intervention package through the existing health system, a parallel cadre of CDAs was constructed. This potentially limits the value of the study in informing scale-up efforts that would likely require a delivery platform that is integrated into existing structures, such as Safe Motherhood Action Groups ${ }^{30}$ or 
Table 3 Impact of the intervention on caregiver-child interaction, child symptoms and child dietary intake

\begin{tabular}{|c|c|c|c|c|c|c|}
\hline & \multirow[b]{2}{*}{ Mean at endline (SD) } & \multirow[b]{2}{*}{ ICC } & \multicolumn{2}{|l|}{ Unadjusted } & \multicolumn{2}{|l|}{ Adjusted $^{*}$} \\
\hline & & & OR (95\% Cl) & p Value & OR (95\% Cl) & p Value \\
\hline \multicolumn{7}{|c|}{ Caregiver-child interaction in last 3 days } \\
\hline \multicolumn{7}{|c|}{ Read books with child } \\
\hline Control & $0.21(0.41)$ & - & - & - & - & - \\
\hline Intervention & $0.50(0.50)$ & 0.07 & 3.71 (2.71 to 5.08$)$ & $<0.0001$ & 3.58 (2.32 to 5.52$)$ & $<0.0001$ \\
\hline \multicolumn{7}{|c|}{ Told stories to child } \\
\hline Control & $0.17(0.38)$ & - & - & - & - & - \\
\hline Intervention & $0.47(0.50)$ & 0.13 & 4.31 (2.44 to 7.62$)$ & $<0.0001$ & 5.61 (3.20 to 9.84$)$ & $<0.0001$ \\
\hline \multicolumn{7}{|c|}{ Sang songs with child } \\
\hline Control & $0.76(0.43)$ & - & - & - & - & - \\
\hline Intervention & $0.90(0.29)$ & 0.03 & $3.02(1.81$ to 5.05$)$ & $<0.0001$ & $3.38(1.85$ to 6.18$)$ & $<0.0001$ \\
\hline \multicolumn{7}{|c|}{ Took child outside the home } \\
\hline Control & $0.52(0.50)$ & - & - & - & - & - \\
\hline Intervention & $0.51(0.50)$ & 0.07 & 0.97 (0.57 to 1.65$)$ & 0.915 & $1.07(0.64$ to 1.80$)$ & 0.790 \\
\hline \multicolumn{7}{|c|}{ Played with child } \\
\hline Control & $0.77(0.42)$ & - & - & - & - & - \\
\hline Intervention & $0.90(0.30)$ & 0.02 & $2.73(1.71$ to 4.34$)$ & $<0.0001$ & 2.59 (1.64 to 4.07$)$ & $<0.0001$ \\
\hline \multicolumn{7}{|c|}{ Named, counted or drew things with child } \\
\hline Control & $0.40(0.49)$ & - & - & - & - & - \\
\hline Intervention & $0.69(0.47)$ & 0.09 & 3.35 (2.23 to 5.01$)$ & $<0.0001$ & 3.58 (2.41 to 5.33$)$ & $<0.0001$ \\
\hline \multicolumn{7}{|c|}{ Child symptoms in the last 2 weeks } \\
\hline \multicolumn{7}{|l|}{ Fever } \\
\hline Control & $0.44(0.50)$ & - & - & - & - & - \\
\hline Intervention & $0.36(0.48)$ & 0.00 & 0.73 (0.52 to 1.02$)$ & 0.069 & $0.68(0.47$ to 0.98$)$ & 0.041 \\
\hline \multicolumn{7}{|l|}{ Diarrhoea } \\
\hline Control & $0.44(0.50)$ & - & - & - & - & - \\
\hline Intervention & $0.35(0.48)$ & 0.03 & $0.71(0.46$ to 1.08$)$ & 0.111 & 0.65 (0.41 to 1.03$)$ & 0.065 \\
\hline \multicolumn{7}{|l|}{ Cough } \\
\hline Control & $0.69(0.46)$ & - & - & - & - & - \\
\hline Intervention & $0.61(0.49)$ & 0.07 & $0.70(0.40$ to 1.24$)$ & 0.221 & 0.63 (0.36 to 1.07$)$ & 0.089 \\
\hline \multicolumn{7}{|c|}{ Child dietary intake in the last 24 hours } \\
\hline \multicolumn{7}{|l|}{ Breastmilk } \\
\hline Control & $0.47(0.50)$ & - & - & - & - & - \\
\hline Intervention & $0.39(0.49)$ & 0.10 & $0.70(0.38$ to 1.28$)$ & 0.245 & $0.66(0.35$ to 1.26$)$ & 0.209 \\
\hline \multicolumn{7}{|l|}{ Meat } \\
\hline Control & $0.49(0.50)$ & - & - & - & - & - \\
\hline Intervention & $0.59(0.49)$ & 0.04 & 1.35 (0.86 to 2.13$)$ & 0.197 & $1.29(0.77$ to 2.14$)$ & 0.331 \\
\hline \multicolumn{7}{|c|}{ Non-meat protein $†$} \\
\hline Control & $0.71(0.45)$ & - & - & - & - & - \\
\hline Intervention & $0.83(0.37)$ & 0.03 & 1.48 (0.96 to 2.27$)$ & 0.075 & $1.43(0.88$ to 2.31$)$ & 0.150 \\
\hline \multicolumn{7}{|c|}{ Breakfast meal (maize) $\ddagger$} \\
\hline Control & $0.33(0.47)$ & - & - & - & - & - \\
\hline Intervention & $0.26(0.44)$ & 0.09 & 0.73 (0.40 to 1.32$)$ & 0.297 & 0.66 (0.37 to 1.19$)$ & 0.170 \\
\hline \multicolumn{7}{|c|}{ Roller meal (maize) } \\
\hline Control & $0.87(0.33)$ & - & - & - & - & - \\
\hline Intervention & $0.90(0.29)$ & 0.01 & 1.36 (0.77 to 2.42$)$ & 0.293 & 1.50 (0.92 to 2.44$)$ & 0.103 \\
\hline
\end{tabular}




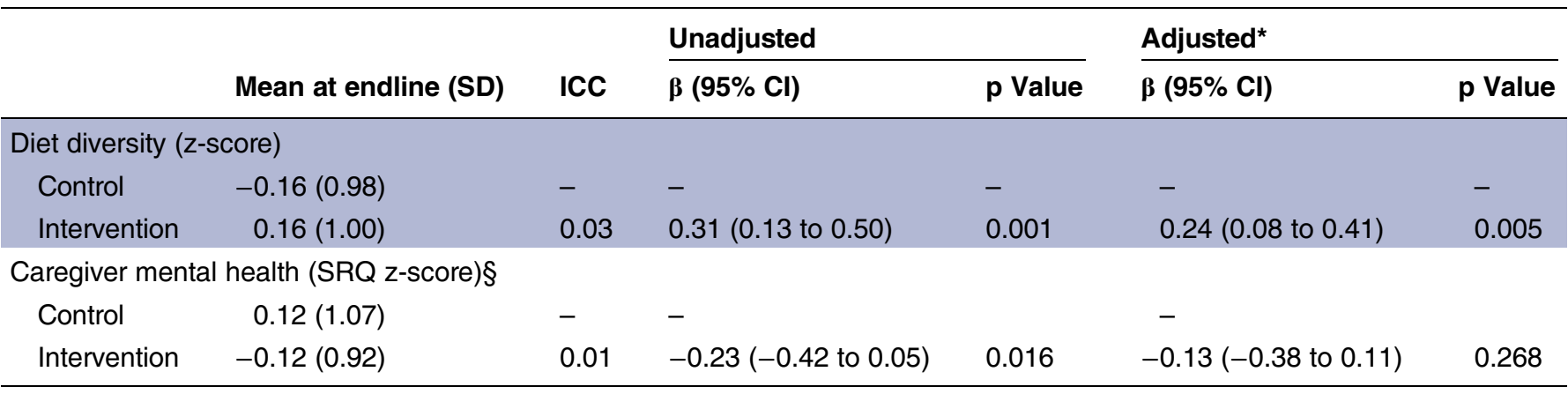

${ }^{*}$ Controlling for: child's age, gender, siblings, baseline HAZ, baseline WAZ, baseline WHZ, baseline motor function Z-score, caregiver's age, education and baseline SRQ z-score, baseline caregiver-child interaction score, household wealth, household inhabitants and gender of household head.

†Non-meat proteins include soy, eggs, nuts and beans.

łBreakfast meal is twice-processed maize, whereas roller meal is once-processed maize. Roller meal has been shown to have higher protein content in part because more of the maize shell is retained.

§Lower SRQ z-score indicates better mental health.

HAZ, height-for-age z-score; ICC, intracluster correlation coefficient; SB, Saving Brains Early Child Development Scale; SRQ, Self-Reporting Questionnaire; WAZ, weight-for-age z-score; WHZ, Weight-for-height z-score.

CHWs delivering integrated community case management. ${ }^{31}$ Second, the intervention package had two components and we cannot identify the independent impact of each component. For this reason, we cannot say whether the behaviour changes we observed were due to household visits by CDAs or group meetings. Third, our measurement of parenting behaviour was largely dependent on self-reports, and parents may have had reasons to misreport their own behaviour, introducing a source of potential bias in our estimates. Finally, and related, the tools used to assess child development may not have been sensitive enough to detect early changes in development. While the NDA tool we used to directly assess children has been validated in a range of lowincome settings ${ }^{25}$ implementation of the tool in rural Zambia was challenging due to the relatively young age and low developmental achievement of the children at endline.

\section{CONCLUSION}

This paper adds to a growing literature that aims to identify effective methods for delivering interventions to improve child development outcomes in diverse settings globally. Community-based delivery of services appears to be feasible in this setting and may be the only means of reaching a large number of households that would not be able to access health centre-based services due to distance and resource barriers. Regular household visits coupled with group meetings appear to positively impact caregiver behaviours. In settings like Zambia, where a substantial proportion of children fail to reach their developmental potential, these interventions hold great promise for improving child health and welfare.

However, improving parental behaviour may not immediately yield large improvements in developmental outcomes; continued and sustained parental and governmental efforts are likely needed to allow children to fully reach their developmental potential. Household resource constraints, in particular, may limit the potential impact of behaviour changes, and complementary government services that ease these constraints, for example food distribution programmes, may be important. Future research should aim to understand caregiver demand for child development services. This will serve as a foundation for developing interventions to stimulate demand when appropriate, and also for designing services to be more responsive to caregiver preferences, a key determinant of successful uptake. In addition, the feasibility, acceptability and cost-effectiveness of various platforms for delivering community-based child development services should continue to be investigated. In some settings, the health system may be best equipped to deliver these services, while in other settings, alternative platforms might be more appropriate.

\section{Author affiliations \\ ${ }^{1}$ Department of Global Health, Boston University School of Public Health, Boston, Massachusetts, USA \\ ${ }^{2}$ Department of Global Health and Population, Harvard TH Chan School of Public Health, Boston, Massachusetts, USA \\ ${ }^{3}$ American Institutes for Research, Washington, District of Columbia, USA \\ ${ }^{4}$ Zambia Center for Applied Health Research and Development, Lusaka, \\ Zambia \\ ${ }^{5}$ Barbara Davis Center for Childhood Diabetes, University of Colorado, Denver, Colorado, USA \\ ${ }^{6}$ Zambia Ministry of Health, Lusaka, Zambia}

\section{Handling editor Sanni Yaya}

Acknowledgements Innovations for Poverty Action managed endline data collection. The authors thank the $10 \mathrm{CDAs}$ who delivered the intervention package and the nurses in charge at study RHCs for their tireless efforts during the project. AZ received salary support from Fogarty International Center of the US National Institutes of Health (R25 TW009340).

Contributors PCR, GF, AZ, GB, SM, VS and DHH contributed to the conception and design of the study. PCR, GF, AZ, BB, GB, CS, SM, VS and $\mathrm{DHH}$ contributed to the organisation of the conduct of the study. PCR, GF, $\mathrm{AZ}, \mathrm{BB}, \mathrm{GB}, \mathrm{CS}$ and $\mathrm{DHH}$ carried out the study, including acquisition of study 
data. PCR, GF, AZ and DHH analysed and interpreted the study data. PCR wrote the first draft of the manuscript. PCR, GF, AZ and DHH contributed important intellectual content to subsequent drafts of the manuscript. All authors have read and approved the final manuscript.

Funding Grand Challenges Canada provided funding for this study through the Saving Brains initiative (0349-03). After the project started, PATH provided additional funding through support provided by UK Aid from the UK Government as part of the Nutrition Embedding Evaluation Program (DFI.1836-672968-GRT). The views expressed do not necessarily reflect the UK Government's official policies.

Disclaimer The funders had no role in study design, data collection and analysis, decision to publish or preparation of the manuscript.

Competing interests None declared

Ethics approval This study was approved by the Institutional Review Board at Boston University and from ERES Converge in Zambia. Additional approvals were obtained from the Zambia Ministry of Health and the Southern Province Medical Office.

Provenance and peer review Not commissioned; externally peer reviewed.

Data sharing statement No additional data are available.

Open Access This is an Open Access article distributed in accordance with the Creative Commons Attribution Non Commercial (CC BY-NC 4.0) license, which permits others to distribute, remix, adapt, build upon this work noncommercially, and license their derivative works on different terms, provided the original work is properly cited and the use is non-commercial. See: http:// creativecommons.org/licenses/by-nc/4.0/

\section{REFERENCES}

1. Stevens GA, Finucane MM, Paciorek CJ, et al. Trends in mild, moderate, and severe stunting and underweight, and progress towards MDG 1 in 141 developing countries: a systematic analysis of population representative data. Lancet 2012;380:824-34

2. Grantham-McGregor S, Cheung YB, Cueto S, et al. Developmental potential in the first 5 years for children in developing countries. Lancet 2007;369:60-70.

3. Walker SP, Wachs TD, Gardner JM, et al. Child development: risk factors for adverse outcomes in developing countries. Lancet 2007;369:145-57.

4. Engle PL, Black MM, Behrman JR, et al. Strategies to avoid the loss of developmental potential in more than $\mathbf{2 0 0}$ million children in the developing world. Lancet 2007;369:229-42.

5. Chilenje Infant Growth, Nutrition and Infection (CIGNIS) Study Team Micronutrient fortification to improve growth and health of maternally HIV-unexposed and exposed Zambian infants: a randomised controlled trial. PLOS ONE 2010;5:e11165.

6. Scharf RJ, DeBoer MD, Guerrant RL. Recent advances in understanding the long-term sequelae of childhood infectious diarrhea. Curr Infect Dis Rep 2014;16:408.

7. Grantham-McGregor SM, Powell CA, Walker SP, et al. Nutritional supplementation, psychosocial stimulation, and mental development of stunted children: the Jamaican study. Lancet 1991;338:1-5.

8. Powell $\mathrm{C}$, Baker-Henningham $\mathrm{H}$, Walker $\mathrm{S}$, et al. Feasibility of integrating early stimulation into primary care for undernourished Jamaican children: cluster randomised controlled trial. $B M J$ 2004;329:89.

9. Singla DR, Kumbakumba E, Aboud FE. Effects of a parenting intervention to address maternal psychological wellbeing and child development and growth in rural Uganda: a community-based, cluster randomised trial. Lancet Glob Health 2015;3:e458-69.

10. Aboud FE, Yousafzai AK. Global health and development in early childhood. Annu Rev Psychol 2015;66:433-57.
11. Yousafzai AK, Rasheed MA, Rizvi A, et al. Effect of integrated responsive stimulation and nutrition interventions in the Lady Health Worker programme in Pakistan on child development, growth, and health outcomes: a cluster-randomised factorial effectiveness trial. Lancet 2014;384:1282-93.

12. Lewycka S, Mwansambo C, Rosato M, et al. Effect of women's groups and volunteer peer counselling on rates of mortality, morbidity, and health behaviours in mothers and children in rural Malawi (MaiMwana): a factorial, cluster-randomised controlled trial. Lancet 2013;381:1721-35.

13. Prost A, Colbourn T, Seward N, et al. Women's groups practising participatory learning and action to improve maternal and newborn health in low-resource settings: a systematic review and meta-analysis. Lancet 2013;381:1736-46.

14. Aboud FE. Evaluation of an early childhood parenting programme in rural Bangladesh. J Health Popul Nutr 2007;25:3-13.

15. Aboud FE, Singla DR, Nahil MI, et al. Effectiveness of a parenting program in Bangladesh to address early childhood health, growth and development. Soc Sci Med 2013;97:250-8.

16. Rao N, Sun J, Pearson V, et al. Is something better than nothing? An evaluation of early childhood programs in Cambodia. Child Dev 2012;83:864-76.

17. Central Statistical Office [Zambia], Ministry of Health [Zambia], and ICF International. Zambia demographic and health survey 2013-14. Rockville, MD, USA: Central Statistical Office, Ministry of Health, and ICF International, 2014.

18. Hamer $\mathrm{DH}$, Herlihy JM, Musokotwane $\mathrm{K}$, et al. Engagement of the community, traditional leaders, and public health system in the design and implementation of a large community-based cluster-randomized trial of umbilical cord care in Zambia. Am J Trop Med Hyg 2015;92:666-72.

19. Nelson CA, Zeanah $\mathrm{CH}$, Fox NA, et al. Cognitive recovery in socially deprived young children: the Bucharest Early Intervention Project. Science 2007;318:1937-40.

20. World Health Organization, United Nations Children's Fund. WHO child growth standards and the identification of severe acute malnutrition in infants and children. Geneva: WHO, 2009

21. Inter-Agency Taskforce on HIV and ECD. The essential package: holistically addressing the needs of young vulnerable children and their caregivers affected by HIV and AIDS. Atlanta: Care International, 2012.

22. World Health Organization, United Nations Children's Fund. Care for child development: improving the care of young children (intervention package). Geneva: World Health Organization, 2012.

23. Jinks C, Jordan K, Croft P. Evaluation of a computer-assisted data entry procedure (including Teleform) for large-scale mailed surveys. Comput Biol Med 2003;33:425-37.

24. World Health Organization. WHO child growth standards: methods and development. Geneva: WHO, 2006.

25. Fernandes M, Stein A, Newton CR, et al. The INTERGROWTH-21st Project neurodevelopment package: a novel method for the multi-dimensional assessment of neurodevelopment in pre-school age children. PLoS ONE 2014;9:e113360.

26. Beusenberg M, Orley J. A user's guide to the self reporting questionnaire (SRQ). Geneva: World Health Organization, 1994.

27. Steyn NP, Nel JH, Nantel G, et al. Food variety and dietary diversity scores in children: are they good indicators of dietary adequacy? Public Health Nutr 2006;9:644-50.

28. United Nations Children's Fund. Multiple indicator cluster surveys (MICS). New York: Unicef, 1995.

29. Victora CG, de Onis M, Hallal PC, et al. Worldwide timing of growth faltering: revisiting implications for interventions. Pediatrics 2010;125:e473-80.

30. Kruk ME, Vail D, Austin-Evelyn K, et al. Evaluation of a maternal health program in Uganda and Zambia finds mixed results on quality of care and satisfaction. Health Aff (Millwood) 2016;35:510-19.

31. Seidenberg PD, Hamer $\mathrm{DH}$, lyer $\mathrm{H}$, et al. Impact of integrated community case management on health-seeking behavior in rural Zambia. Am J Trop Med Hyg 2012;87:105-10. 